\title{
Abundance Gradients and Substructures in Disks
}

\author{
K. C. Freeman \\ Research School of Astronomy \& Astrophysics \\ Mount Stromlo Observatory \\ The Australian National University
}

\begin{abstract}
I will first discuss abundance gradients in the Milky Way and nearby disk galaxies, then the problem of substructures in the Galactic Disk, and finally some new opportunities for investigating substructure in disks.
\end{abstract}

Keywords. abundances, star clusters, disks, kinematics and dynamics

\section{Abundance Gradients}

In this section, I will discuss abundance gradients as observed in the Milky Way, M31, and the two similar late-type disk systems M33 and NGC 300. But first some comments on the truncation of disks.

The disk of M33 shows a classical truncation behaviour: out to a radius of about 35 arcmin, the surface brightness profile follows closely the usual exponential decline with radius. Then, at a surface brightness of about $24 \mathrm{I}$ mag $\operatorname{arcsec}^{-2}$, the slope steepens and this steeper exponential continues to the limit of the measurable surface brightness (about $30 \mathrm{mag} \operatorname{arcsec}^{-2}$ ) (Ferguson et al. 2007). This kind of truncation was long believed to be ubiquitous, but it is now known that the surface brightness distribution in the outer regions of disks can take other forms. For example NGC 300, which has a similar appearance and absolute magnitude to M33, continues its exponential decline without any change in slope for about 10 scalelengths to the limit of the surface photometry, again at about $30 \mathrm{mag} \operatorname{arcsec}^{-2}$ (Bland-Hawthorn et al. 2005). Erwin et al. (2005) identified three kinds of outer disk morphology: those which continue as a single exponential (type I, like NGC 300), those which steepen at large radii (type II, the classical truncations, like M33), and those in which the surface brightness profile in the outer disk flattens in slope at large radii (type III).

The classical type II truncations are not understood. Various explanations have been proposed, including angular momentum redistribution by bars and spiral waves, the effect of the star formation threshold at low surface density, the hierarchical accretion process, and bombardment by dark matter subhalos (de Jong et al. 2007). Roškar et al. (2008) made an SPH simulation of disk formation from cooling gas in an isolated dark halo, including star formation and feedback, which shows the type II break in the surface brightness profile. The break is seeded by a rapid radial decrease in the surface density of cool gas: the break becomes visible within 1 Gyr and gradually moves outwards as the disk grows. The outer (steeper) exponential is fed by secularly redistributed stars from the inner regions, via transient disturbances and the Sellwood \& Binney (2002) orbit-swapping process, so its stars are relatively old (see Fig. 1).

The Galactic disk shows an abundance gradient, seen very clearly in the abundances of the Galactic cepheids (Luck et al. 2006). The mean abundance of these relatively young 


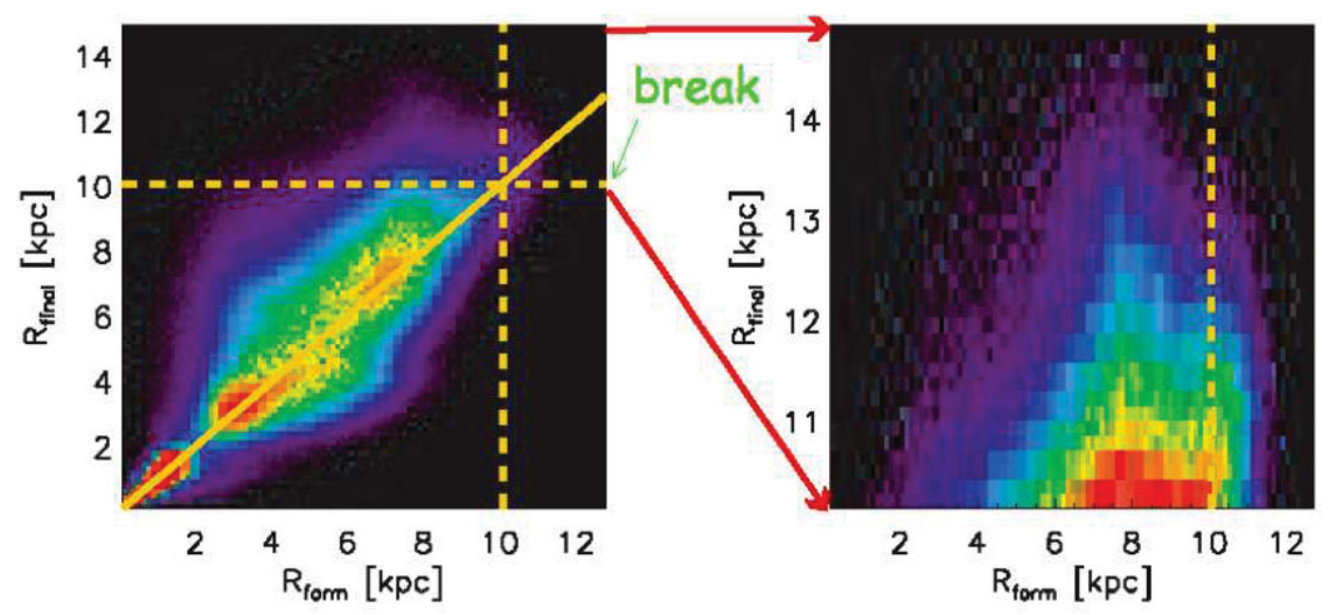

Figure 1. The redistribution of disk stars by the Sellwood \& Binney (2002) mechanism: the density distribution of stars in the plane of formation radius $R_{\text {form }}$ against final radius $R_{\text {final }}$. The label "break" shows the location of the break in the final surface brightness profile. The right panel shows an expanded view of the region $\mathrm{R}_{\text {final }}>10 \mathrm{kpc}$. Adapted from Roškar et al. (2008).

stars falls from $[\mathrm{Fe} / \mathrm{H}]=+0.2$ at radius $\mathrm{R}=5 \mathrm{kpc}$ to about -0.5 at $15 \mathrm{kpc}$. It is not a simple axisymmetric gradient, however: near the sun, a tongue of relatively metal-rich stars extends out into the $l=90-180^{\circ}$ quadrant, with a corresponding tongue of metalpoor stars extending inwards in the third quadrant (see Fig. 2). This asymmetry may be related to noncircular motions induced by the spiral structure.

The Galactic abundance gradient is seen also for older star clusters and red giants (Yong \& Carney 2005, Carney \& Yong 2005). For the open clusters, with ages between about 1 and 5 Gyr, the abundance gradient appears to bottom out at a radius of about $12 \mathrm{kpc}$ and and abundance of -0.5 (M31 shows a similar outer plateau in its abundance gradient, beginning at a radius of $15 \mathrm{kpc}$ and at the same abundance level: Worthey et al. 2004). These older stars in the outer Galactic disk are $\alpha$-enhanced, with $[\alpha / \mathrm{Fe}]=$ +0.2 , indicating fairly rapid chemical evolution of the gas in the outer disk from which they formed (unlike the stars of similar age in the solar neighborhood). Comparing the abundance gradients and $\alpha$-enhancement for these older stars with those for the younger cepheids, it appears that the abundance gradient and the $[\alpha / \mathrm{Fe}]$ gradients have flattened with time, tending towards the solar values.

If the outer gas has evolved in situ, then the $\alpha$-enrichment of the 5-Gyr old clusters in the outer galaxy suggests that their chemical evolution was quick: i.e. star formation in the outer disk has been going on for only about 6 Gyr. This is an internal version of down-sizing, depending on surface density rather than total mass. Not only is the star formation timescale longer in the outer Galaxy (Chiappini et al. 2001) but star formation and chemical evolution started later.

A recent study of red giants in the outer regions of NGC 300 (Vlajić et al. 2008) shows an apparent reversal of the abundance gradient in the outer regions. This reversal could be associated with gas accretion. Alternatively, it may result from the orbit-swapping phenomenon mentioned above, with the outermost disk populated by stars scattered out from the more metal-rich inner regions. The similar galaxy M33 shows a clear abundance gradient in its inner exponential component: this gradient appears to flatten in the outer disk where its surface brightness gradient has steepened (Barker et al. 2007). As in the 
Milky Way, there is evidence that the abundance gradient in M33 has flattened with time: the gradient appears to be flatter for the HII regions and young stars than for the somewhat older planetary nebulae and red giants (Magrini et al. 2007).

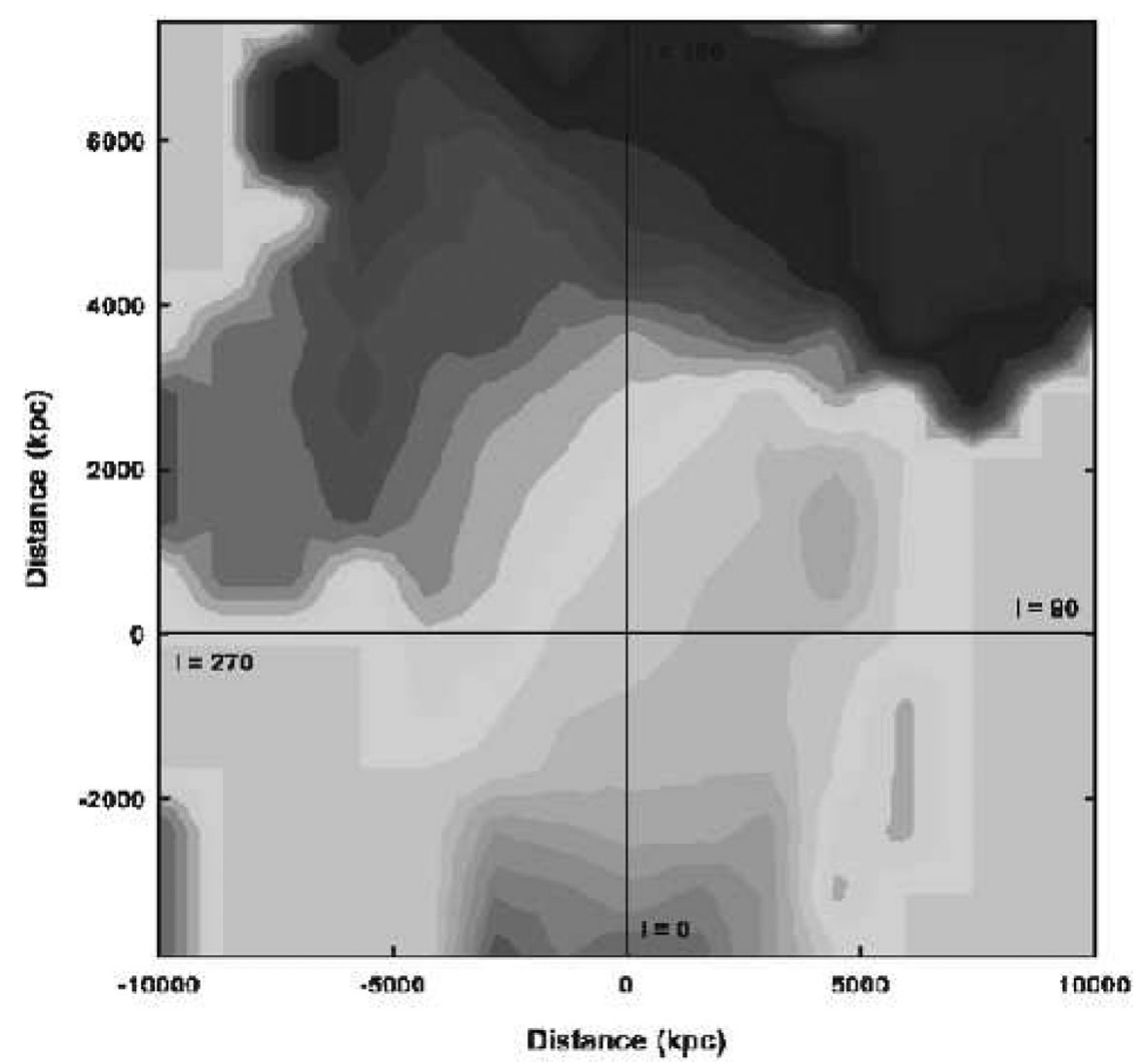

Figure 2. The abundance distribution of cepheids on the galactic plane. The origin is at the sun and the y-axis points towards the anticenter. In the mean, the abundance decreases with radius, but a tongue of metal-richer cepheids extends out into the second quadrant. Adapted from Luck et al. (2006).

In the vertical direction, photometric abundances for a very large sample of Galactic FG stars from the SDSS show that the abundance distribution in the Galactic disk and thick disk is stratified parallel to the Galactic plane for heights $|Z|>1 \mathrm{kpc}$. The Monoceros stream appears as a metal-rich feature extended in $Z$ at a radius of about 15 kpc (Ivezić et al. 2008). The vertical abundance gradient from SDSS agrees well with an independent estimate from thin disk clump giants (Soubiran et al. 2008).

Kinematics for a subsample of the SDSS stars at a mean height $Z \sim 1.1 \mathrm{kpc}$ show that the azimuthal lag velocity $\left\langle v_{\phi}\right\rangle$ is constant at about $40 \mathrm{~km} \mathrm{~s}^{-1}$ and is uncorrelated with abundance for stars in the abundance range $-0.4>[\mathrm{Fe} / \mathrm{H}]>-1$ (Ivezić et al. 2008). This is unexpected; in the solar neighborhood the correlation of disk kinematics with abundance has been long known: e.g. Strömgren (1986). 
To summarize this section on abundance gradients:

- The outer disks of M31, M33, NGC 300 and the Galaxy include a component that is at least several Gyr old

- The abundance gradients in the outer disks of M31 and the Galaxy bottom out at large $\mathrm{R}$, at $[\mathrm{Fe} / \mathrm{H}]=-0.5$. The gradient in $\mathrm{M} 33$ also flattens at large $\mathrm{R}$ and the gradient in NGC 300 reverses in the outer disk

- The abundance gradients in the Galaxy and M33 appear to flatten with time

- The older stars of the outer Galactic disk are $\alpha$-enhanced, indicating rapid chemical evolution. This $\alpha$-enhancement is less for the younger stars in the outer disk.

What processes are important for determining the properties of outer disks: gas accretion, tidal effects, orbit swapping ...?

\section{Substructures in the Disk}

Substructures in the disk are mostly difficult to see in configuration space. Exceptions are the substructures seen in the SDSS data (Jurić et al. 2008, Ivezić et al. 2008) which include the Monoceros feature and two other less promonent disk substructures seen in maps of residuals from a smooth model of the SDSS star counts.

Most disk substructures are identified in (U,V) velocity space as stellar moving groups: ( $\mathrm{U}$ and $\mathrm{V}$ are the stellar velocity component relative to the local standard of rest (LSR) towards $l=0$ and $l=90^{\circ}$ respectively). The groups are made up of nearby stars, all around us, which share common U,V motions. The concept of these moving groups goes back to Kapteyn and Eggen. The major nearby moving groups can be seen very clearly in Dehnen's (1999) analysis of Hipparcos motions for nearby stars; it shows the Sirius, Hyades and Pleiades moving groups which dominate the local stellar velocity distribution at low U,V velocities, and also the higher velocity Hercules group (see Fig. 3).

Although the moving groups are not yet well understood, it appears that they are not all produced in the same way:

- some are associated with dynamical resonances (Galactic bar, spiral structure) so their chemical abundance distribution is expected to be broad and fairly typical of the nearby thin and thick disks. The Hercules group is a likely example of a resonance group.

- some are the debris of star-forming aggregates in the disk. The HR1614 group is an example (see below). Such groups may be chemically homogeneous and are potentially very useful for reconstructing the star forming events which built up the Galactic disk.

- Others may be debris of infalling objects, as seen in CDM simulations like those of Abadi et al. (2003). Navarro et al. (2004) proposed that the thick disk Arcturus Group may be an example of this kind of stellar moving group.

The HR1614 group is an example of a group which appears to be the dispersed debris of an old star forming event. Its age is about $2 \mathrm{Gyr}$ and its metallicity $[\mathrm{Fe} / \mathrm{H}]=+0.2$. It was studied by Feltzing \& Holmberg (2000) who argued for its reality as a relic group. De Silva et al. (2007) measured very precise chemical abundances for many elements in the stars of the HR1614 group, and found a very small spread in the abundances. This is in marked contrast to the stars of the Hercules group studied by Bensby et al. (2007) who found that the Hercules stars appear chemically to be a fairly typical sample of field stars of the thin and thick disk. This supports the view that the Hercules group is dynamically associated with a resonance that affects a wide range of disk stars.

The nature of the Hyades-Sirius complex, which is the dominant feature of the U,V distribution for the nearby stars, remains uncertain. The ages of these stars appears to be less than 1 Gyr: is this substructure the dispersing remnant of a star-forming event, 


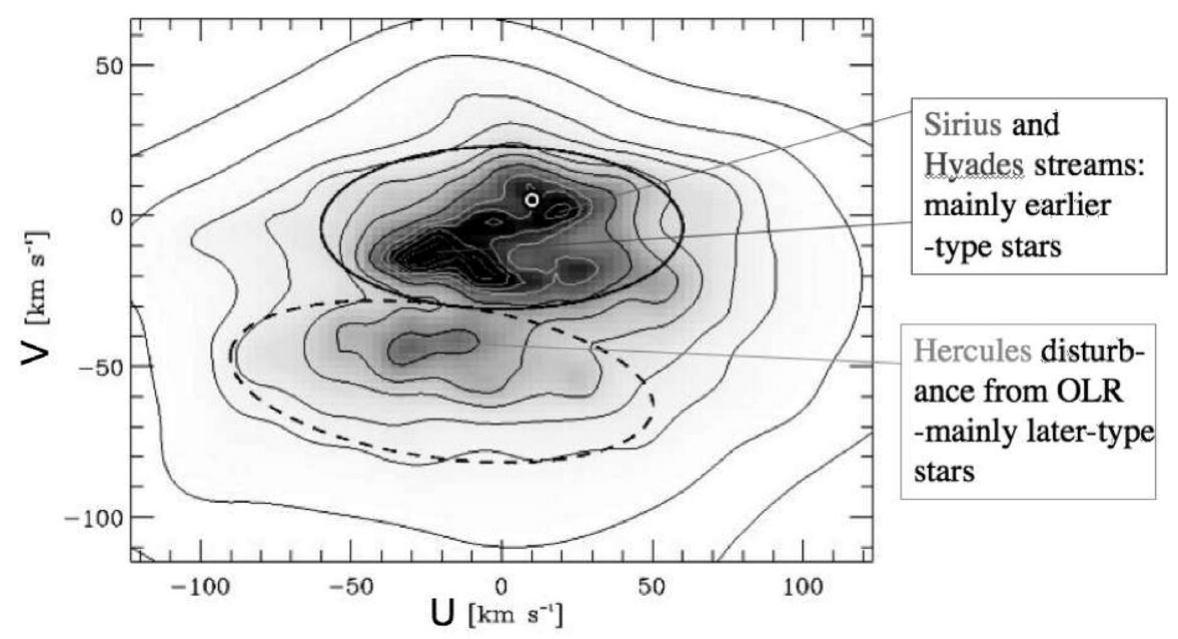

Figure 3. (U,V) distribution of Hipparcos stars, showing the major stellar moving groups which dominate the (U,V) distribution for the nearby stars. Adapted from Dehnen (1999).

or another example of a resonance feature. Quillen \& Minchev (2005) showed how Lindblad resonances with spiral density waves can generate velocity space structures that have a morphology at least qualitatively like that of the Hyades-Sirius complex. If the Hyades-Sirius groups are dispersing aggregates passing through the solar neighborhood, then their stars should lie on Lindblad dispersion orbits and therefore on well-defined tracks in the (longitude - radial velocity) plane. Wilson (1988) made a high resolution spectroscopic study of this kind for a large sample of $\mathrm{K}$ giants near the galactic plane, and found that the dispersion orbit loci are seen but only in a narrow $[\mathrm{Fe} / \mathrm{H}]$ range (similar to the abundance of the Hyades cluster), as would be expected for dispersing star forming events (see Fig. 4). On the other hand, Famaey et al. (2008) argue that the stars of the Hyades-Sirius complex include stars with a wide range of ages and are therefore most likely a resonance phenomenon.

To summarize this section on moving groups:

- The HR1614 moving group (age 2 Gyr) appears to be the relic of an old dispersed star-forming event. This is good: we can use such groups to reconstruct the galactic disk. But how has this moving group maintained its dynamical identity for 2 Gyr against the effects of disk heating?

- The Hercules group is chemically inhomogeneous and is probably just a sample of disk stars swept together in velocity space by local dynamical resonances. Mary Williams' new data on the thick disk Arcturus group (Williams, 2008) indicates that it too is probably a resonance phonomenon.

- Many dispersed aggregates will not be recognizable kinematically, because their dynamical identity has been lost. Many kinematical structures are not dispersed aggregates: they are manifestations of resonances with the gravitational fields of bar and spiral wave.

- New resonant substructures are being found in (U,V) space by several authors (eg Fuchs et al., Williams et al.). It seems likely that most of the kinematically defined substructures found in the future will turn out to be associated with resonances. While these are dynamically interesting, they are not much use for probing fossil debris of ancient star forming events. Chemical signatures will probably be more useful for finding fossil debris. 
$-0.1>[\mathrm{Fe} / \mathrm{H}]>-0.2$

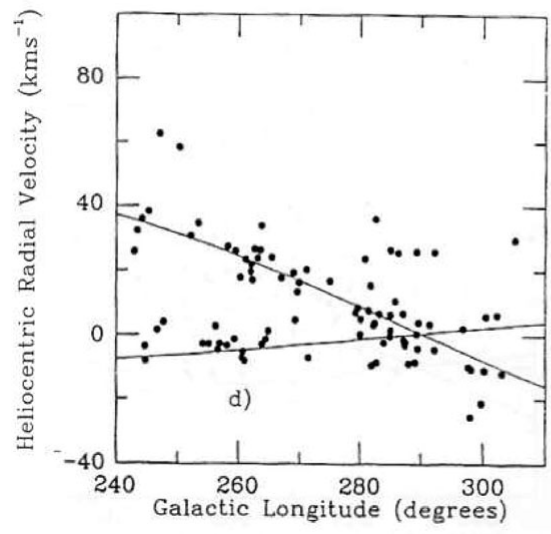

$[\mathrm{Fe} / \mathrm{H}]<-0.25$

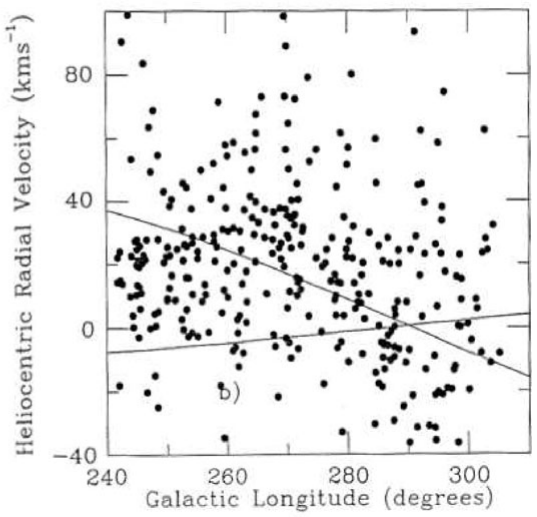

Figure 4. Distribution of bright $\mathrm{K}$ giants at low galactic latitude in the radial velocity vs galactic longitude plane. The solid curves show the loci of predicted Lindblad dispersion orbits calculated for the observed (U,V) motions of the Hyades and Sirius moving groups. Stars in the abundance range $-0.1>[\mathrm{Fe} / \mathrm{H}]>-0.2$ appear to be clustered around the dispersion orbit loci. Stars with $[\mathrm{Fe} / \mathrm{H}]<-0.25$ show no such clustering tendency. Adapted from Wilson (1988).

\section{New Opportunities for Studying Substructure}

- The detailed abundance pattern of the stars in a dispersed star-forming aggregate reflect the chemical evolution of the gas from which the stars formed. These element abundance patterns can be used in chemical tagging, to detect fossil substructure that is no longer dynamically recognizable because of disk heating and orbit-swapping (Freeman \& Bland-Hawthorn 2002).

- the hydrostatics of the HI provide a way to detect extended gravitating dark substructures in the disk (Kalberla et al. 2007, O'Brien et al. 2008).

Chemical Tagging: For chemical tagging to work, a few conditions need to be satisfied. Stars must form in large aggregates, and this is believed to be true. The aggregates must be chemically homogeneous, and they must have unique chemical signatures, defined by several elements which do not vary in lockstep from one aggregate to another. Also, a sufficient spread in abundance from aggregate to aggregate is needed, so that their chemical signatures can be distinguished within the accuracy achievable observationally (about 0.05 dex differentially in the element abundances). Testing these chemical conditions was the goal of Gayandhi De Silva's thesis on open cluster and moving group abundances, and they appear to be true (see Fig. 5 and her paper in this volume).

Chemical tagging requires a large high resolution stellar spectroscopic survey of about one million stars (Bland-Hawthorn \& Freeman 2004). Such large surveys will be feasible in the relatively near future, with two new instruments that are planned. WFMOS is a proposed joint project by Subaru and Gemini. The current concept has 1000 fibers over a 1.5-degree field, feeding spectrometers with a resolution of about 30,000. This instrument would enable a large survey with a signal-to-noise ratio of about 100 per resolution element, down to $V \sim 17$. HERMES on the AAT has 400 fibers in a 2-degree field. The resolution is again about 30,000. A large survey is planned down to $V \sim 14$; this magnitude limit matches the fiber density to the typical Galactic stellar density. HERMES has already had its concept design review and is scheduled to begin operation in 2012 . 

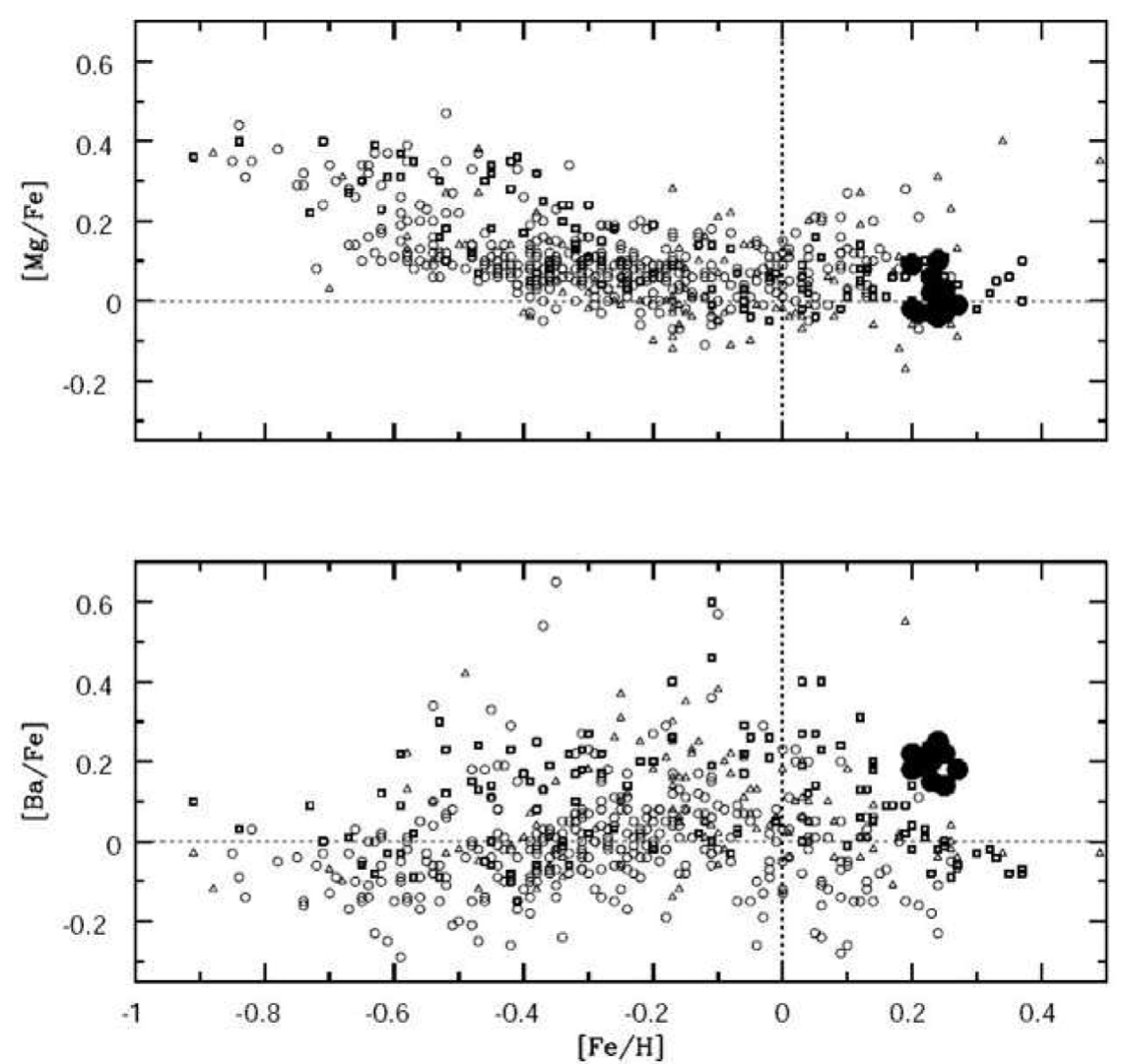

Figure 5. Chemical properties of stars in the HR1614 moving group. The upper panel shows $[\mathrm{Mg} / \mathrm{Fe}]$ vs $[\mathrm{Fe} / \mathrm{H}]$ abundances for stars of the HR1614 group (large filled circles) and for field stars (small open circles). The lower panel is similar, for $[\mathrm{Ba} / \mathrm{Fe}]$. The chemical homogeneity of the HR1614 group stars is evident. Adapted from De Silva et al. (2007).

WFMOS and HERMES have an outstanding synergy with GAIA, which will provide precision astrometry for about a billion stars. GAIA is at its best for the relatively bright stars which will be targeted with these two spectrometers. For example, at the V-magnitude limit of 14 planned for the HERMES survey, the expected parallax and proper motion uncertainties are $\sigma_{\pi}=10 \mu$ as and $\sigma_{\mu}=10 \mu \mathrm{as} \mathrm{yr}^{-1}$ respectively. These uncertainties correspond to $1 \%$ distance errors at a distance of $1 \mathrm{kpc}$, and transverse velocity errors of $0.7 \mathrm{~km} \mathrm{~s}^{-1}$ at a distance of $15 \mathrm{kpc}$. The combination of GAIA and HERMES will give accurate distances, element abundances and 3D velocities for most of the survey stars, and isochrone ages for many. It will be possible to construct accurate color-(absolute magnitude) diagrams for the survey stars, providing an independent check that chemically tagged groups of stars do indeed have common ages.

The Hydrostatics of the HI Layer in Disk Galaxies: The hydrostatics of the HI layer in disk galaxies provides a potentially powerful technique to measure the potential gradient $\partial \Phi / \partial z$ to complement the radial potential gradient $\partial \Phi / \partial R$ from rotation data. In combination, the two potential gradients can be used to

- estimate the shape of the dark halo (e.g. Olling \& Merrifield 2000) 
- settle the maximum disk controversy, because $\partial \Phi / \partial z$ in the inner disk near the galactic plane comes mainly from the stellar disk

- identify concentrations of dark matter in galactic disks, such as might be generated by the accretion of satellite galaxies in circularized orbits

It seems likely that HI hydrostatics will be used more in future, as high spatial resolution HI data with adequate signal-to-noise become available for disk galaxies. To illustrate the power of HI hydrostatics, Kalberla et al. (2007) used the HI flaring of the Galactic $\mathrm{HI}$ layer to explore the Galactic potential. The HI density data is extends to $\mathrm{R}=40 \mathrm{kpc}$ and $\mathrm{z}=20 \mathrm{kpc}$, but little is known about how the HI velocity dispersion changes with height above the galactic plane. They had to assume an isothermal velocity dispersion for the Galactic HI; their conclusions depend on this assumption. To fit the observed HI distribution, they required several dark components, in addition to the known luminous components:

- the usual dark halo with a mass of about $1.8 \times 10^{12} \mathrm{M}_{\odot}$

- a dark disk with a scale length $h_{R}=8 \mathrm{kpc}$, a scale height $h_{z}=4 \mathrm{kpc}$ and a mass of about $2 \times 10^{11} \mathrm{M}_{\odot}$ (although the mass of this dark disk is about 4 times larger than the mass of the stellar disk, it would probably have escaped detection via stellar kinematics).

- a dark ring at radius $R=13-20 \mathrm{kpc}$, with mass of $2.5 \times 10^{10} \mathrm{M}_{\odot}$, which may be associated with the Monoceros stellar ring.

To use the hydrostatics of the HI layer, one needs to assume that the HI layer is in equilibrium and that its velocity dispersion is isotropic. These assumptions are plausible, at least for some disk galaxies. In addition, for a secure analysis, one needs to know (i) the density distribution $\rho(R, z)$ of the HI layer as a function of $R$ and $z$, and (ii) how the rotation $V(R, z)$ and velocity dispersion $\sigma(R, z)$ of the HI depend on $R$ and $z$. Using newly developed techniques, it is now possible to measure $\rho(R, z), V(R, z)$ and $\sigma(R, z)$ for the HI in relatively nearby edge-on galaxies (O'Brien et al. 2009).

\section{References}

Abadi, M. et al. 2003, ApJ, 597, 21

Barker, M. et al. 2007. ApJ, 133, 1138

Bensby,T. et al. 2007, ApJ, 655, L89

Bland-Hawthorn, J. \& Freeman, K. 2004, PASA, 21, 110

Bland-Hawthorn, J. et al. 2005, ApJ, 629, 239

Carney, B. \& Yong, D. 2005, AJ, 130, 597

Chiappini, C. et al. 2001, ApJ, 554, 1044

Dehnen, W. 1999, ApJ, 524, L35

de Jong, R. et al. 2007, , 667, L49

De Silva, G. et al. 2007, AJ, 133, 694

Erwin, P. et al. 2005, ApJ, 626, L81

Famaey, B. et al. 2008, A\&A 483,453

Feltzing, S. \& Holmberg, J. 2000, A\& A, 357, 153

Ferguson, A. et al. 2007, in "Island Universes", Astrophysics and Space Science Proceedings, Springer, 239

Freeman, K. \& Bland-Hawthorn, J 2002, ARAA, 40, 487

Ivezić, Z. et al. 2008, astro-ph/0804.3850

Jurić, M. et al. 2008, ApJ, 673, 864

Kalberla, P. et al. 2007, A\&SA, 469, 511

Luck, R.E. et al. 2006, $A J, 132,902$

Magrini, L. et al. 2007, A\&BA, 470, 843

Navarro, K. et al. 2004, ApJ, 601, L43 
O'Brien, J. et al. 2009, in preparation

Olling, J. \& Merrifield, M. 2000, MNRAS, 311, 361

Quillen, A. \& Minchev, I. 2005, AJ, 130, 576

Roškar, R. et al. 2008, ApJ, 675, L65

Sellwood, J. \& Binney, J. 2002, MNRAS, 336, 785

Soubiran, C. et al. 2008, A\&BA, 480, 91

Strömgren, B. 1986, in "The Galaxy", NATO ASI Series, (Reidel) Vol 207. Ed

G. Gilmore \& R. Carswell, p 229

Vlajić, M. et al. 2008, in this volume

Williams, M. 2008, in this volume

Wilson, G. 1988, ANU thesis

Yong, D. \& Carney, B. 2005, AJ

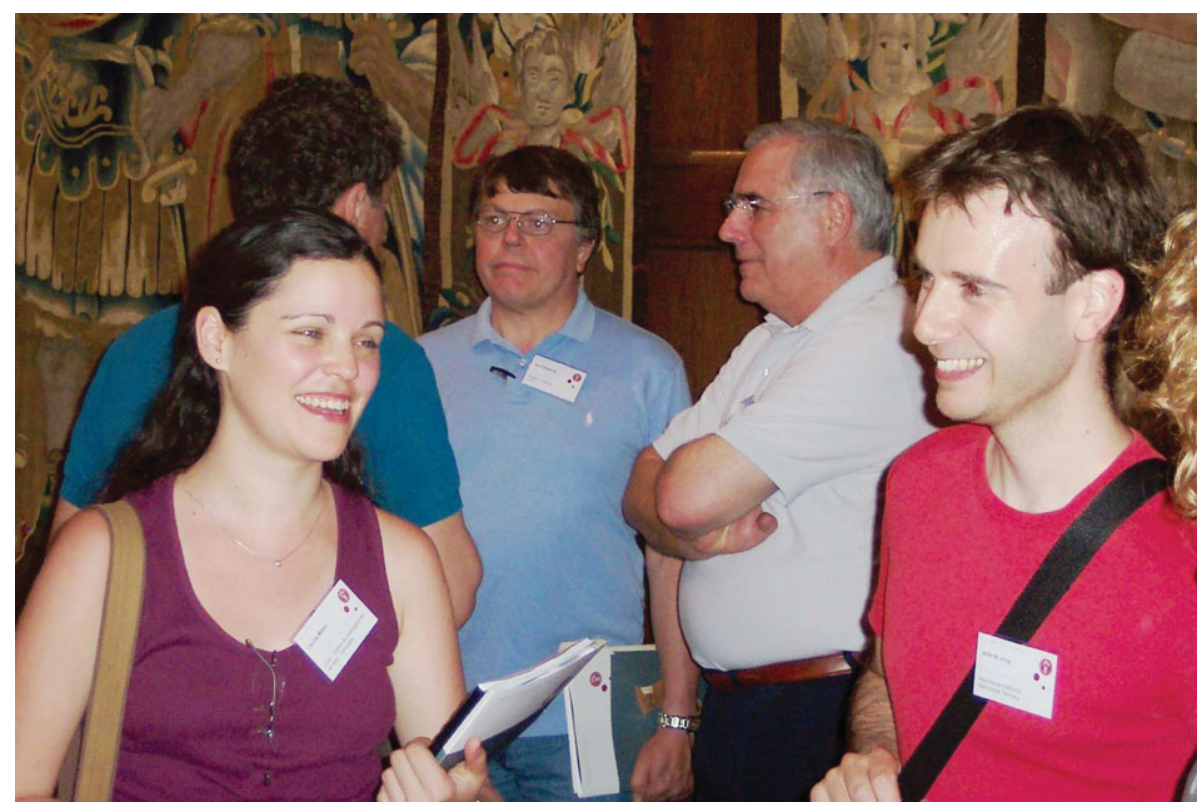

Cecilia Mateu and Jelte de Jong enjoying the welcome reception. In the background, Simon White, Jerry Sellwood and Leo Blitz are deciding the fate of the Galaxy. 


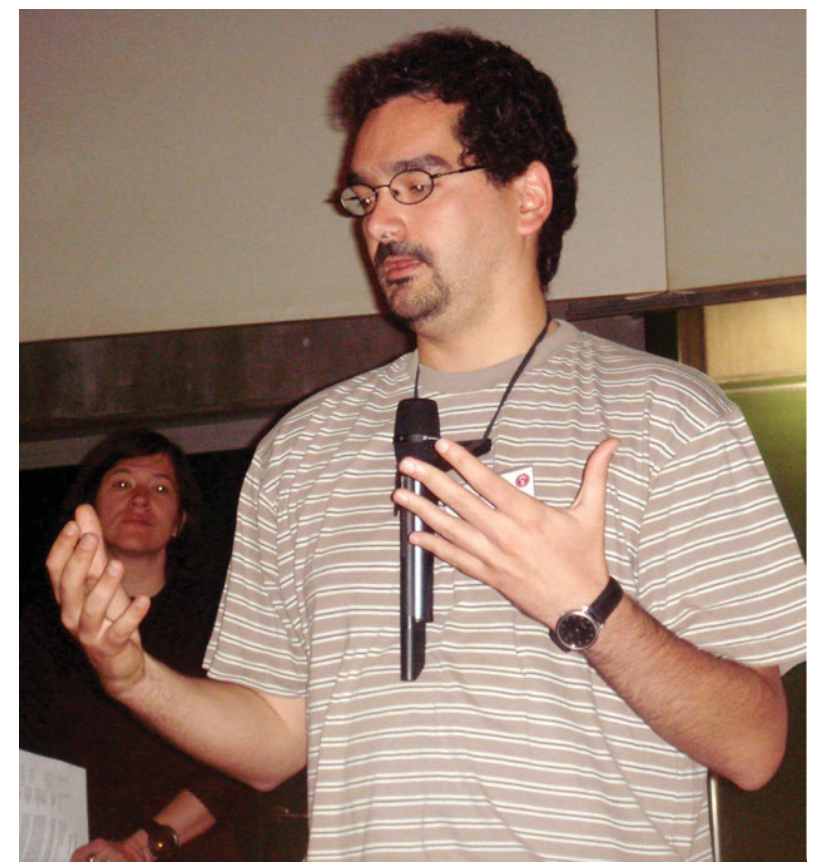

Ignacio Trujillo explaining a point during his presentation.

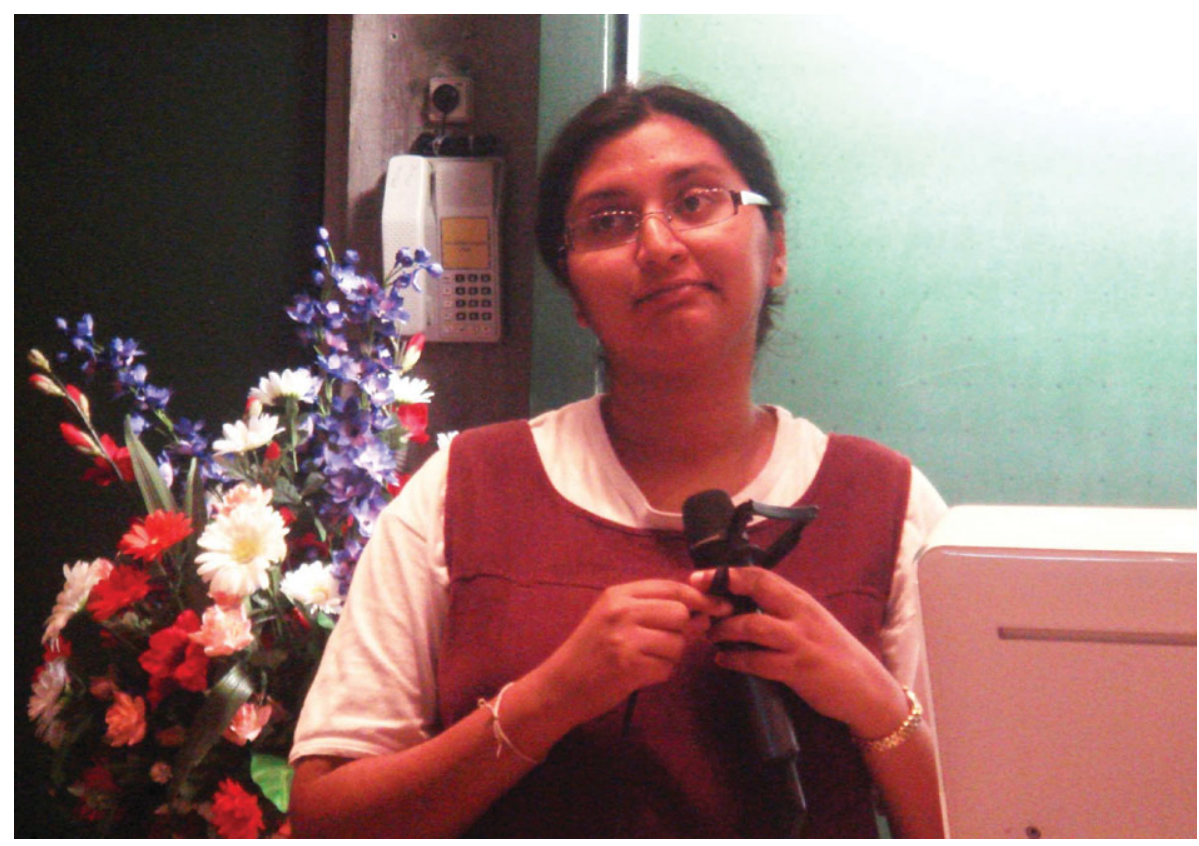

Gayandhi De Silva fielding a question after her talk. 